\title{
Soft-core Coulomb potentials and Heun's differential equation
}

\author{
Richard L. Hall ${ }^{1}$, Nasser Saad $^{2}$, and K. D. Sen ${ }^{3}$ \\ 1 Department of Mathematics and Statistics, Concordia University, \\ 1455 de Maisonneuve Boulevard West, Montréal, Québec, Canada H3G 1M8 \\ 2 Department of Mathematics and Statistics, University of Prince Edward Island, \\ 550 University Avenue, Charlottetown, PEI, Canada C1A 4P3. and \\ 3 School of Chemistry, University of Hyderabad 500046, India ${ }^{*}$
}

\begin{abstract}
Schrödinger's equation with the attractive potential $V(r)=-Z /\left(r^{q}+\beta^{q}\right)^{\frac{1}{q}}, Z>0, \beta>0, q \geq 1$, is shown, for general values of the parameters $Z$ and $\beta$, to be reducible to the confluent Heun equation in the case $q=1$, and to the generalized Heun equation in case $q=2$. In a formulation with correct asymptotics, the eigenstates are specified a priori up to an unknown factor. In certain special cases this factor becomes a polynomial. The Asymptotic Iteration Method is used either to find the polynomial factor and the associated eigenvalue explicitly, or to construct accurate approximations for them. Detail solutions for both cases are provided.
\end{abstract}

PACS: 31.15.-p, 31.10.+z; 36.10.Ee; 36.20.Kd; 03.65.Ge.

Keywords: Soft-core Coulomb potential, asymptotic iteration method, confluent Heun equation, generalized Heun equation, quasi-exact solutions.

\section{INTRODUCTION}

Analytic wave functions corresponding to solutions of Schrödinger's time-independent equation $H \Psi=E \Psi$ for standard quantum-mechanical systems, such as the hydrogen atom and the harmonic oscillator, have usually been constructed by transforming the original equations in to a differential equation whose solutions are known in terms of certain special functions. Indeed the general solutions of the resulting Kummer equation corresponding to such model systems, as expressed in the form of confluent hypergeometric functions, have led to a deeper understanding of these systems, under both free [1, 2] and spatially confined conditions [3]. In this paper we consider the model quantum system defined by the Hamiltonian (in atomic units $m=\hbar=e=1$ )

$$
H=-\frac{1}{2} \Delta+V_{q}(r), \quad V_{q}(r)=-\frac{Z}{\left(r^{q}+\beta^{q}\right)^{\frac{1}{q}}} .
$$

The potential $V_{q}(r)$ represents a family of soft-core (truncated) Coulomb potentials, which are useful as model potentials in atomic and molecular physics. The bound states are obtained in terms of three potential parameters: the coupling $Z>0$, the cut-off parameter $\beta>0$, and the power parameter $q \geq 1$. The cases $q=1$ and 2 are of special physical significance $4,\left[5,66,7,8,6,10,11,12\right.$. The potential $V_{1}$ represents the potential due to a smeared charge and is useful in describing mesonic atoms. The potential $V_{2}$ is similar to the shape of the potential due to a finite nucleus and experienced by the muon in a muonic atom. Extensive applications of the soft-core Coulomb potential, $V_{2}$, have been made through model calculations corresponding to the interaction of intense laser fields with atoms [13, 14, 15, 16, 17, 18]. The parameter $\beta$ can be related to the strength of the laser field, with the range $\beta=20-40$ covering the experimental laser field strengths [13].

In an earlier paper [19], we have carried out a general analysis of the characteristic features of the energies and wave functions of the complete family of soft Coulomb potentials defined by $V_{q}$. The Schrödinger operator $H$ is bounded below. This may be shown immediately by an application of the operator inequality [20, 21] $-\Delta>1 /\left(4 r^{2}\right)$, which yields the general spectral bound

$$
E>\min _{r>0}\left[\frac{P^{2}}{2 r^{2}}+V_{q}(r)\right], \quad P=\frac{1}{2} .
$$

We showed in Ref. [19] that general upper and lower estimates for all the discrete eigenvalues can be expressed in this form (for suitable $P$ ), with the aid of envelope theory [22, 23, 24, 25]. If the exact eigenvalues of $H$ are written

\footnotetext{
*Electronic address: rhall@mathstat.concordia.ca Electronic address: nsaad@upei.ca Electronic address: sensc@uohyd.ernet.in
} 
$E(Z, \beta, q)$, then these spectral functions are monotone in each parameter, decreasing in $Z$ and $q$, and increasing in $\beta$. Thus

$$
\frac{\partial E}{\partial Z}<0, \quad \frac{\partial E}{\partial \beta}>0, \quad \text { and } \quad \frac{\partial E}{\partial q}<0 .
$$

Moreover, the following general scaling law is obeyed:

$$
E(Z, \beta, q)=Z^{2} E(1, Z \beta, q)=\frac{1}{\beta^{2}} E(Z \beta, 1, q) .
$$

In the limit as $q \rightarrow \infty$, the potential descends to the cut-off Coulomb potential $V_{\infty}$ given by

$$
\lim _{q \rightarrow \infty} V(r)=V_{\infty}(r)= \begin{cases}-\frac{Z}{\beta}, & \text { if } r<\beta \\ -\frac{Z}{r}, & \text { if } r \geq \beta .\end{cases}
$$

This much is known for the whole class of problems.

Mehta and Patil [4] have presented analytical solutions for the $s$-state eigenvalues corresponding to the $V_{1}$ potential. Patil [5] has also discussed the analyticity of the scattering phase shifts for two particles interacting through the potentials $V_{q}$ with $q=1$ and $q=2$. Singh et al [6] have reported a large number of eigenvalues for the states $1 s$ to $4 f$ corresponding to $V_{1}$ and $V_{2}$ for a fixed value of $Z$; these values were obtained by the numerical solution of Eq.(1) for $Z=1$. The scaling law (2) extends their application to other values of $Z$. Exact bound-state solutions of $V_{1}$ have been considered earlier [7, 8, 9, 10], in which only a limited number of states with a specific choice of $\ell=0 \ldots 3$ have been treated. To our knowledge, no such study for $q \geq 2$ has been reported so far.

The principal results of the present paper are as follows. We show that Schrödinger's equation for the two physically important cases, $q=1$ and $q=2$, can both be solved analytically, in terms of the Heun differential equation and the generalized Heun equation, respectively. Interestingly, in a suitable formulation, we show that exact solutions exist in which a factor in the wave function becomes a polynomial. It is shown that such solutions, and the corresponding exact eigenvalues, can be found explicitly by the use of an iterative method called the Asymptotic Iteration Method (AIM) [26, 27, 28, 29, 30, 31, 32, 33, 34, 35]. In cases where such polynomial solutions are not possible, AIM can also be used to construct approximate wave functions and eigenvalues. We summarize AIM in the next section, and we formulate the eigenvalue problem for general $q \geq 1$ in section 3 . In sections 4 and 5 we discuss in detail the cases $q=1$ and $q=2$, respectively, providing tables of exact analytical solutions, where possible, and approximations in other cases.

\section{THE ASYMPTOTIC ITERATION METHOD}

The asymptotic iteration method (AIM) was first introduced as a technique capable of overcoming the computation complexity of solving Schrödinger's equation with singular potentials. It was first used as an approximation method for computing both eigenvalues and eigenstates, with the aid of a computer-algebra system. Because of its explicit iterative structure, the the method has subsequently been employed very effectively to obtain exact analytic solutions to many problems involving linear differential equations, especially those presented by non-relativistic and relativistic quantum mechanics. Recently, some progress has been made also in adapting the method to non-linear differential equations [36]. Perhaps because of its success in finding exact solutions, the strength of AIM as an approximation method has not been emphasized. For useful approximate solutions, the iteration sequence must be started with a suitable form for the wave function, and also a starting position $r=r_{0}$. If these two seeds are well chosen, then either the sequence stops at an exact solution, or a non-ending iteration sequence is generated, which can then be terminated to yield a good approximation. Asymptotics usually provides a reliable guide to the initial form for the wave function; the starting point $r_{0}$ is then chosen so as to stabilize the resulting iteration process. The present work illustrates the effectiveness of AIM in finding both exact and approximate solutions of quantum-mechanical problems generated by soft-core Coulomb potentials. We shall now outline the method. Consider a differential equation of the form

$$
y^{\prime \prime}=\lambda_{0}(r) y^{\prime}+s_{0}(r) y, \quad\left({ }^{\prime}=\frac{d}{d r}\right)
$$

where $\lambda_{0}(r)$ and $s_{0}(r)$ are $C^{\infty}$-differentiable functions. A key feature of this method is to note the invariant structure of the right-hand side of (3) under further differentiation. Indeed, if we differentiate (3) with respect to $r$, we obtain

$$
y^{\prime \prime \prime}=\lambda_{1} y^{\prime}+s_{1} y
$$


where $\lambda_{1}=\lambda_{0}^{\prime}+s_{0}+\lambda_{0}^{2}$ and $s_{1}=s_{0}^{\prime}+s_{0} \lambda_{0}$. If we find the second derivative of equation (3), we obtain

$$
y^{(4)}=\lambda_{2} y^{\prime}+s_{2} y
$$

where $\lambda_{2}=\lambda_{1}^{\prime}+s_{1}+\lambda_{0} \lambda_{1}$ and $s_{2}=s_{1}^{\prime}+s_{0} \lambda_{1}$. Thus, for $(n+1)^{t h}$ and $(n+2)^{t h}$ derivative of (3), $n=1,2, \ldots$, we have

$$
y^{(n+1)}=\lambda_{n-1} y^{\prime}+s_{n-1} y
$$

and

$$
y^{(n+2)}=\lambda_{n} y^{\prime}+s_{n} y
$$

respectively, where

$$
\lambda_{n}=\lambda_{n-1}^{\prime}+s_{n-1}+\lambda_{0} \lambda_{n-1} \quad \text { and } \quad s_{n}=s_{n-1}^{\prime}+s_{0} \lambda_{n-1} .
$$

From (6) and (7) we have

$$
\lambda_{n} y^{(n+1)}-\lambda_{n-1} y^{(n+2)}=\delta_{n} y \quad \text { where } \quad \delta_{n}=\lambda_{n} s_{n-1}-\lambda_{n-1} s_{n} .
$$

Clearly, from (9) if $y$, the solution of (3), is a polynomial of degree $n$, then $\delta_{n} \equiv 0$. Further, if $\delta_{n}=0$, then $\delta_{n^{\prime}}=0$ for all $n^{\prime} \geq n$. In an earlier paper [26] we proved the principal theorem of the Asymptotic Iteration Method (AIM), namely

Theorem 1: Given $\lambda_{0}$ and $s_{0}$ in $C^{\infty}(a, b)$, the differential equation (3) has the general solution

$$
y(r)=\exp \left(-\int^{r} \alpha(t) d t\right)\left[C_{2}+C_{1} \int^{r} \exp \left(\int^{t}\left(\lambda_{0}(\tau)+2 \alpha(\tau)\right) d \tau\right) d t\right]
$$

if for some $n>0$

$$
\frac{s_{n}}{\lambda_{n}}=\frac{s_{n-1}}{\lambda_{n-1}} \equiv \alpha
$$

For a given potential, the radial Schrödinger equation is converted to the form of equation (3). Then, $s_{0}(r)$ and $\lambda_{0}(r)$ are determined and the $s_{n}(r)$ and $\lambda_{n}(r)$ parameters are calculated from the recurrence relations given by equation (8). The energy eigenvalues are obtained from the roots of the termination condition of the method in equation (11), or equivalently,

$$
\delta_{n}(E ; r)=\lambda_{n}(E ; r) s_{n-1}(E ; r)-\lambda_{n-1}(E ; r) s_{n}(E ; r) \equiv 0, \quad n=1,2, \ldots
$$

where $n$ represents the iteration number. For the exactly solvable problems, the energy eigenvalues are obtained immediately from the equation

$$
\delta_{n}(E ; r)=\delta_{n}(E) \equiv 0,
$$

and very often the eigenvalue index is the same as the iteration number $n$. For problems that do not have exact solutions expressible in the form chosen, the approximations for the eigenvalues are taken to be the roots of this same equation for large iteration number.

\section{SOFT-CORE COULOMB POTENTIALS $V_{q}(r)$}

In atomic units, the radial Schrödinger equation for the potential $V_{q}(r)$ reads

$$
\left[-\frac{1}{2} \frac{d^{2}}{d r^{2}}+\frac{l(l+1)}{2 r^{2}}-\frac{Z}{\left(r^{q}+\beta^{q}\right)^{\frac{1}{q}}}\right] \psi=E \psi .
$$


We may assume the solution of equation (13), which vanishes at the origin and at infinity, is

$$
\psi(r)=r^{l+1} e^{-k\left(r^{q}+\beta^{q}\right)^{\frac{1}{q}}} f\left(\left(r^{q}+\beta^{q}\right)^{\frac{1}{q}}\right)
$$

Straightforward computation shows that $f\left(\left(r^{q}+\beta^{q}\right)^{\frac{1}{q}}\right)$ is a solution of the second-order homogeneous linear differential equation

$$
\begin{aligned}
r^{2 q-2}\left(r^{q}+\beta^{q}\right)^{\frac{2}{q}-2} f^{\prime \prime}\left(\left(r^{q}+\beta^{q}\right)^{\frac{1}{q}}\right)+ & {\left[(q-1) \beta^{q} r^{q-2}\left(r^{q}+\beta^{q}\right)^{\frac{1}{q}-2}\right.} \\
+ & \left.2 \nu r^{q-2}\left(r^{q}+\beta^{q}\right)^{\frac{1}{q}-1}-2 k r^{2 q-2}\left(r^{q}+\beta^{q}\right)^{\frac{2}{q}-2}\right] f^{\prime}\left(\left(r^{q}+\beta^{q}\right)^{\frac{1}{q}}\right) \\
+ & {\left[-k(q-1) \beta^{q} r^{q-2}\left(r^{q}+\beta^{q}\right)^{\frac{1}{q}-2}-2 k \nu r^{q-2}\left(r^{q}+\beta^{q}\right)^{\frac{1}{q}-1}\right.} \\
& \left.+k^{2} r^{2 q-2}\left(r^{q}+\beta^{q}\right)^{\frac{2}{q}-2}+2 Z\left(r^{q}+\beta^{q}\right)^{-\frac{1}{q}}+2 E\right] f\left(\left(r^{q}+\beta^{q}\right)^{\frac{1}{q}}\right)=0 .
\end{aligned}
$$

where, for simplicity, we write $\nu=l+1$. From equation (15), we obtain, after some simplification,

$$
\begin{aligned}
f^{\prime \prime}\left(\left(r^{q}+\beta^{q}\right)^{\frac{1}{q}}\right) & +\left[\frac{(q-1) \beta^{q}}{r^{q}\left(r^{q}+\beta^{q}\right)^{\frac{1}{q}}}+\frac{2 \nu}{r^{q}\left(r^{q}+\beta^{q}\right)^{\frac{1}{q}}}-2 k\right] f^{\prime}\left(\left(r^{q}+\beta^{q}\right)^{\frac{1}{q}}\right) \\
& +\left[-\frac{k(q-1) \beta^{q}}{r^{q}\left(r^{q}+\beta^{q}\right)^{\frac{1}{q}}}-\frac{2 k \nu}{r^{q}\left(r^{q}+\beta^{q}\right)^{\frac{1}{q}-1}}+k^{2}+\frac{2 Z}{r^{2 q-2}\left(r^{q}+\beta^{q}\right)^{\frac{3}{q}-2}}+\frac{2 E}{r^{2 q-2}\left(r^{q}+\beta^{q}\right)^{\frac{2}{q}-2}}\right] f\left(\left(r^{q}+\beta^{q}\right)^{\frac{1}{q}}\right)=0
\end{aligned}
$$

If we denote $\chi=\left(r^{q}+\beta^{q}\right)^{\frac{1}{q}}$, we may now write (16) in a more compact form, as

$$
\begin{aligned}
f^{\prime \prime}(\chi) & +\left[\frac{(q-1) \beta^{q}}{\chi\left(\chi^{q}-\beta^{q}\right)}+\frac{2 \nu}{\chi^{1-q}\left(\chi^{q}-\beta^{q}\right)}-2 k\right] f^{\prime}(\chi) \\
& +\left[-\frac{k(q-1) \beta^{q}}{\chi\left(\chi^{q}-\beta^{q}\right)}-\frac{2 k \nu}{\chi^{1-q}\left(\chi^{q}-\beta^{q}\right)}+k^{2}+\frac{2 Z}{\chi^{3-2 q}\left(\chi^{q}-\beta^{q}\right)^{2-\frac{2}{q}}}+\frac{2 E}{\chi^{2-2 q}\left(\chi^{q}-\beta^{q}\right)^{2-\frac{2}{q}}}\right] f(\chi)=0
\end{aligned}
$$

where differentiation is now with respect to $\chi$. This equation is now in a form suitable for the application of AIM with:

$$
\left\{\begin{array}{l}
\lambda_{0}(r) \equiv-\frac{(q-1) \beta^{q}}{\chi\left(\chi^{q}-\beta^{q}\right)}-\frac{2 \nu}{\chi^{1-q}\left(\chi^{q}-\beta^{q}\right)}+2 k \\
s_{0}(r) \equiv \frac{k(q-1) \beta^{q}}{\chi\left(\chi^{q}-\beta^{q}\right)}+\frac{2 k \nu}{\chi^{1-q}\left(\chi^{q}-\beta^{q}\right)}-k^{2}-\frac{2 Z}{\chi^{3-2 q}\left(\chi^{q}-\beta^{q}\right)^{2-\frac{2}{q}}}-\frac{2 E}{\chi^{2-2 q}\left(\chi^{q}-\beta^{q}\right)^{2-\frac{2}{q}}} .
\end{array}\right.
$$

In the next two sections we shall study the most important cases for physical applications, namely $q=1$ and $q=2$.

\section{BOUND STATES OF SHIFTED COULOMB POTENTIAL $V_{1}(r)=-Z /(r+\beta)$}

The problem of determining the energy eigenvalues and eigenstates of the shifted Coulomb potential

$$
V_{1}(r)=-\frac{Z}{r+\beta}, \quad \beta>0
$$

has been of some interest in the past. As mentioned in the introduction, the potential may serve as an approximation to the potential due to a smeared charge distribution, rather than a point charge, and may be appropriate for describing mesonic atoms [37]. Using equation (14), the solution of Schrödinger equation with the shifted Coulomb potential $V_{1}(r)$ is

$$
\psi(r)=r^{l+1} e^{-k \chi} f(\chi), \quad \chi=r+\beta,
$$


where $f(\chi)$ is the solution of differential equation

$$
f^{\prime \prime}(\chi)+\left[\frac{2 \nu}{\chi-\beta}-2 k\right] f^{\prime}(\chi)+\left[-\frac{2 k \nu}{\chi-\beta}+k^{2}+\frac{2 Z}{\chi}+2 E\right] f(\chi)=0
$$

or, for $k^{2}=-2 E$, we have

$$
f^{\prime \prime}(\chi)+\left[\frac{2 \nu}{\chi-\beta}-2 k\right] f^{\prime}(\chi)+\left[-\frac{2 k \nu}{\chi-\beta}+\frac{2 Z}{\chi}\right] f(\chi)=0 .
$$

By comparing this equation with equation (18), we can easily write the initial $\lambda_{0}(r)$ and $s_{0}(r)$ values,

$$
\left\{\begin{array}{l}
\lambda_{0}(r) \equiv-\frac{2 \nu}{\chi-\beta}+2 k \\
s_{0}(r) \equiv \frac{2 k \nu}{\chi-\beta}-\frac{2 Z}{\chi}
\end{array}\right.
$$

By means of AIM sequences, Eq. (8), we may calculate $\lambda_{n}(r)$ and $s_{n}(r)$. Equation (12) then gives

$$
\delta_{1} \equiv 0 \Rightarrow(Z-k \nu)(Z-k \nu-k) \chi^{2}-2 Z \beta(Z-k \nu-k) \chi+Z b(Z \beta-k \beta-\nu) \equiv 0 .
$$

From this equation, for $k=Z /(\nu+1)$, we have a condition on the parameter $\beta$, namely $Z \beta=\nu+1$. In general, we have in terms of the iteration number $n$ (or the degree of the polynomial solution $f(\chi)$ of (22) )

$$
\delta_{n} \equiv 0 \Rightarrow k=\frac{Z}{\nu+n}, \quad n=1,2, \ldots,
$$

with conditions on the parameter $\beta$ reported earlier [19]. For other values of $k$ and $\beta$, not necessary obeying these conditions, the eigenvalues are then computed by means of the termination condition (12), namely $\delta_{n}(r ; E)=0$. As we mentioned above, the computation of the eigenvalues by means of (12) should be independent of the choice of $r$. However, for certain values of $r_{0}$, we may encounter oscillations of the computed roots and values that seem to diverge, presumably owing to rounding and computational errors in the algorithms used. In practice, this problem is avoided by re-choosing $r_{0}$. In Table 【 we show some results of these calculation obtained by means of the computer algebra system Maple. This environment allows numerical calculation of the roots of equation (12) with arbitrary chosen precision. In order to accelerate the computation we have written our own root-finding algorithm instead of using the default procedure Solve of Maple. In Table I we also report the iteration number along with the initial $r_{0}$ used to obtain the eigenvalues accurate to the number of decimal places recorded. For much smaller values of the parameter $\beta$, a large number of iterations are usually needed, along with careful adjustment of $r_{0}$.

\section{A. Analytic solutions for $V_{1}(r)=-Z /(r+\beta)$ and the confluent Heun equation}

It is interesting that the polynomial solutions of $f(\chi)$ of equation (22) are completely captured by AIM through the relations governed by $k$ and $\beta$ reported in Table 1 . In other words, no other polynomial solutions are possible. In order to confirm this claim, we now express the solution of the differential equation (22) in terms of the confluent Heun function. From Appendix I, it is clear that the differential equation (22) is a special case of the confluent Heun differential equation (69). Indeed with simple transformation $z=\chi / \beta$, equation (22) can be written as

$$
\frac{d^{2} f}{d z^{2}}+\left[\frac{2 \nu}{z-1}-2 k \beta\right] \frac{d f}{d z}+\left[-\frac{2 k \nu \beta}{z-1}+\frac{2 Z \beta}{z}\right] f=0 .
$$

Further, the transformation $z=1-t$ yields

$$
\frac{d^{2} f}{d t^{2}}+\left[\frac{2 \nu}{t}+2 k \beta\right] \frac{d f}{d t}+\left[\frac{2 k \nu \beta}{t}-\frac{2 Z \beta}{t-1}\right] f=0,
$$

which is clearly a special case of (69). Exact solutions are given (see equations (70) and (71)) by

$$
f(t)=H e\left(2 k \beta, 2 v-1,-1,-2 Z \beta, \frac{1}{2}, t\right) .
$$


TABLE I: Approximate solutions of the radial Schrödinger equation (13) for $q=1$.

\begin{tabular}{|c|c|c|c|c|}
\hline$\beta$ & $l$ & Energy & $N$ & $r_{0}$ \\
\hline \multirow{2}{*}{200} & 1 & -0.0031695328 & 17 & 210 \\
\hline & 3 & -0.0024982718 & 17 & 210 \\
\hline \multirow[t]{2}{*}{100} & 0 & -0.0067420767 & 17 & 120 \\
\hline & 3 & -0.0041687844 & 13 & 150 \\
\hline \multirow[t]{4}{*}{50} & 0 & -0.0121946926 & 16 & 75 \\
\hline & 1 & -0.0097175884 & 18 & 100 \\
\hline & 2 & -0.0079627963 & 13 & 100 \\
\hline & 3 & -0.0066438818 & 16 & 110 \\
\hline 35 & 3 & -0.0082686807 & 14 & 90 \\
\hline \multirow[t]{4}{*}{20} & 0 & -0.0256699378 & 38 & 65 \\
\hline & 1 & -0.0188462206 & 30 & 65 \\
\hline & 2 & -0.0143872079 & 22 & 65 \\
\hline & 3 & -0.0112782933 & 22 & 75 \\
\hline \multirow[t]{4}{*}{10} & 0 & -0.0434387193 & 56 & 45 \\
\hline & 1 & -0.0294465157 & 49 & 55 \\
\hline & 2 & -0.0210243016 & 43 & 65 \\
\hline & 3 & -0.0155766001 & 42 & 75 \\
\hline
\end{tabular}

For the polynomial solutions of $f(t)$, the parameters of $H e\left(2 k \beta, 2 v-1,-1,-2 Z \beta, \frac{1}{2}, t\right)$ have to obey the conditions of equation (72). The first condition of (72) immediately yields

$$
k=\frac{Z}{\nu+N}
$$

where $N$ is the degree of the polynomial solution. This is in complete agreement with the results of AIM as given by (25). While the second condition of (72) now yields the conditions under which these polynomial solutions are possible, namely,

$$
\Delta_{N+1}(2 k \nu \beta)=0
$$

where $\Delta_{N+1}(2 k \nu \beta)$ is given by means of the tri-diagonal determinant of Table $\amalg$. Since $t=1-z=1-\frac{\chi}{\beta}=1-\frac{r+\beta}{\beta}=$ $-\frac{r}{\beta}$, the Taylor series expansion $f_{N}(t)$ becomes

$$
f_{N}(t) \equiv H e\left(\frac{2 Z \beta}{N+\nu}, 2 \nu-1,-1,-2 Z \beta, \frac{1}{2},-\frac{r}{\beta}\right)=\sum_{n=0}^{\infty} \nu_{n}\left(\frac{2 Z \beta}{N+\nu}, 2 \nu-1,-1,-2 Z \beta, \frac{1}{2}\right)\left(-\frac{r}{\beta}\right)^{n} .
$$

Therefore, we have

$$
f_{1}(r)=1+\frac{Z r}{1+\nu}
$$

for

$$
\Delta_{2}\left(\frac{2 Z \nu \beta}{\nu+1}\right) \equiv Z \beta-\nu-1=0
$$


and

$$
f_{2}(r)=1+\frac{Z r}{2+\nu}+\frac{Z\left(-\nu^{2}+(Z \beta-4) \nu+Z \beta-4\right) r^{2}}{\beta(\nu+2)^{2}(2 \nu+1)}
$$

if

$$
\Delta_{3}\left(\frac{2 Z \nu \beta}{\nu+2}\right) \equiv Z^{2}(\nu+1) \beta^{2}-3 Z(\nu+2)(\nu+1) \beta+(2 \nu+1)(\nu+2)^{2}=0
$$

Further

$$
\begin{aligned}
f_{3}(r) & =1+\frac{Z r}{3+\nu}+\frac{Z\left(-\nu^{2}+(Z \beta-6) \nu+Z \beta-9\right) r^{2}}{\beta(\nu+3)^{2}(2 \nu+1)} \\
& +\frac{Z\left(2 \nu^{4}+(19-3 Z \beta) \nu^{3}+\left(Z^{2} \beta^{2}-21 Z \beta+63\right) \nu^{2}+\left(3 Z^{2} \beta^{2}-45 Z \beta+81\right) \nu+2 Z^{2} \beta^{2}-27 Z \beta+27\right) r^{3}}{\beta^{2}(6 \nu+3)(\nu+3)^{3}(\nu+1)}
\end{aligned}
$$

if

$$
\begin{aligned}
\Delta_{4}\left(\frac{2 Z \nu \beta}{\nu+3}\right) & \equiv Z^{3}(\nu+2)(\nu+1) \beta^{3}-6 Z^{2}(\nu+3)(\nu+2)(\nu+1) \beta^{2}+Z\left(11 \nu^{2}+28 \nu+15\right)(\nu+3)^{2} \beta \\
& -3(\nu+1)(\nu+3)^{3}(2 \nu+1)=0
\end{aligned}
$$

and

$$
\begin{aligned}
f_{4}(r) & =1+\frac{Z r}{4+\nu}+\frac{Z\left(-\nu^{2}+(Z \beta-8) \nu+Z \beta-16\right) r^{2}}{\beta(\nu+4)^{2}(2 \nu+1)} \\
& +\frac{Z\left(2 \nu^{4}+(25-3 Z \beta) \nu^{3}+\left(Z^{2} \beta^{2}-27 Z \beta+108\right) \nu^{2}+\left(3 Z^{2} \beta^{2}-72 Z \beta+176\right) \nu+2 Z^{2} \beta^{2}-48 Z \beta+64\right) t^{3}}{\beta^{2}(6 \nu+3)(\nu+4)^{3}(\nu+1)} \\
& +\frac{Z r^{4}}{6 \beta^{3}(\nu+4)^{4}(2 \nu+1)(\nu+1)(2 \nu+3)}\left(\left(-6 \nu^{6}+(11 Z \beta-105) \nu^{5}+\left(-6 Z^{2} \beta^{2}+163 Z \beta-723\right) \nu^{4}+\left(918 Z \beta+Z^{3} \beta^{3}\right.\right.\right. \\
& \left.-2448-66 Z^{2} \beta^{2}\right) \nu^{3}+\left(-252 Z^{2} \beta^{2}-4128+2408 Z \beta+6 Z^{3} \beta^{3}\right) \nu^{2}+\left(-3072+2848 Z \beta-384 Z^{2} \beta^{2}+11 Z^{3} \beta^{3}\right) \nu \\
& \left.\left.-768+1152 Z \beta+6 Z^{3} \beta^{3}-192 Z^{2} \beta^{2}\right)\right)
\end{aligned}
$$

if

$$
\begin{aligned}
\Delta_{5}\left(\frac{2 Z \nu \beta}{\nu+4}\right) & \equiv Z^{4}(\nu+3)(\nu+2)(\nu+1) \beta^{4}-10 Z^{3}(\nu+4)(\nu+3)(\nu+2)(\nu+1) \beta^{3}+Z^{2}\left(35 \nu^{3}+195 \nu^{2}+328 \nu+162\right)(\nu+4)^{2} \beta^{2} \\
& -Z\left(50 \nu^{3}+231 \nu^{2}+313 \nu+126\right)(\nu+4)^{3} \beta+6(2 \nu+3)(2 n+1)(\nu+1)(\nu+4)^{4}=0
\end{aligned}
$$

Similarly, for the higher polynomials, in general,

$$
\Delta_{N+1}(2 k \nu \beta) \equiv 0
$$

where $\Delta_{N+1}(2 k \nu \beta)$ is given by Table

All the $N+1$ roots of this determinant are real and distinct [38]. It may be possible in future to obtain such results by studying the correspondence between the iteration details of AIM and the structure of the resulting tri-diagonal determinant.

\section{BOUND STATES FOR THE SOFT-CORE COULOMB POTENTIAL $V_{2}(r)=-Z / \sqrt{r^{2}+\beta^{2}}$}

For $q=2$ and $\nu=0$, we have using Eq.(17)

$$
f^{\prime \prime}(\chi)+\left[\frac{\beta^{2}}{\chi\left(\chi^{2}-\beta^{2}\right)}+\frac{2 \nu \chi}{\chi^{2}-\beta^{2}}-2 k\right] f^{\prime}(\chi)+\left[-\frac{k \beta^{2}}{\chi\left(\chi^{2}-\beta^{2}\right)}-\frac{2 k \nu \chi}{\chi^{2}-\beta^{2}}+k^{2}+\frac{2 Z \chi}{\chi^{2}-\beta^{2}}+\frac{2 E \chi^{2}}{\chi^{2}-\beta^{2}}\right] f(\chi)=0 .
$$


TABLE II: The determinant $\Delta_{N+1}(2 Z \beta)$. Here $k=Z /(N+\nu)$

\begin{tabular}{|ccccccc}
$2 k \nu \beta$ & $2 \nu$ & 0 & $\ldots$ & 0 & 0 & 0 \\
$2 N k \beta$ & $2 k \nu \beta-(2 \nu-2 k \beta)$ & $2(2 \nu+1)$ & $\ldots$ & 0 & 0 & 0 \\
0 & $2(N-1) k \beta$ & $2 k \nu \beta-2(2 \nu+1-2 k \beta)$ & $\ldots$ & 0 & $\vdots$ & 0 \\
$\vdots$ & $\vdots$ & $\vdots$ & $\ddots$ & $\vdots$ & $(N-1)(N+2 \nu-2)$ & 0 \\
0 & 0 & 0 & $\ldots$ & $2 k \nu \beta-(N-2)(N+2 \nu-2 k \beta-3)$ & \\
0 & 0 & 0 & $\ldots$ & $4 k \beta$ & $2 k \nu \beta-(N-1)(N+2 \nu-2 k \beta-2)$ & $N(N+2 \nu-1)$ \\
0 & 0 & 0 & $\ldots$ & 0 & $2 k \beta$ & $2 k \nu \beta-N(N+2 \nu+2 k \beta-1)$
\end{tabular} \mid

From this equation we have

$$
\left(1-\beta^{2} \chi^{-2}\right) f^{\prime \prime}(\chi)+\left[\beta^{2} \chi^{-3}-2 k+2 k \beta^{2} \chi^{-2}\right] f^{\prime}(\chi)+\left[-k \beta^{2} \chi^{-3}+k^{2}+2 Z \chi^{-1}+2 E-k^{2} \beta^{2} \chi^{-2}\right] f(\chi)=0,
$$

as given earlier by Liu \& Clark [17] for $-2 E=k^{2}$. For the purpose of applying AIM, we may note for $\nu=l+1$ and

$$
2 E=-k^{2}
$$

that

$$
f^{\prime \prime}(\chi)=\left[2 k-\frac{\beta^{2}}{\chi\left(\chi^{2}-\beta^{2}\right)}-\frac{2 \nu \chi}{\chi^{2}-\beta^{2}}\right] f^{\prime}(\chi)+\left[\frac{k \beta^{2}}{\chi\left(\chi^{2}-\beta^{2}\right)}+\frac{2 k \nu \chi}{\chi^{2}-\beta^{2}}+\frac{k^{2} \beta^{2}}{\chi^{2}-\beta^{2}}-\frac{2 Z \chi}{\chi^{2}-\beta^{2}}\right] f(\chi) .
$$

This equation can be further simplified, yielding

or

$$
f^{\prime \prime}(\chi)=\left[2 k+\frac{1}{\chi}-\frac{(2 v+1) \chi}{\chi^{2}-\beta^{2}}\right] f^{\prime}(\chi)+\left[-\frac{k}{\chi}+\frac{((2 \nu+1) k-2 Z) \chi+k^{2} \beta^{2}}{\chi^{2}-\beta^{2}}\right] f(\chi)
$$

$$
f^{\prime \prime}(\chi)=\left[2 k+\frac{1}{\chi}-\frac{(v+1 / 2)}{\chi-\beta}-\frac{(v+1 / 2)}{\chi+\beta}\right] f^{\prime}(\chi)+\left[\frac{(\nu+1 / 2) k-Z+k^{2} \beta / 2}{\chi-\beta}+\frac{(\nu+1 / 2) k-Z-k^{2} \beta / 2}{\chi+\beta}-\frac{k}{\chi}\right] f(\chi) \text {. }
$$

Thus we may now apply AIM with

$$
\left\{\begin{array}{l}
\lambda_{0}=2 k+\frac{1}{\chi}-\frac{(v+1 / 2)}{\chi-\beta}-\frac{(v+1 / 2)}{\chi+\beta} \\
s_{0}=\frac{(\nu+1 / 2) k-Z+k^{2} \beta / 2}{\chi-\beta}+\frac{(\nu+1 / 2) k-Z-k^{2} \beta / 2}{\chi+\beta}-\frac{k}{\chi} .
\end{array}\right.
$$

Using AIM sequence (8) we can thus produce a set of exact polynomial solutions given by

$$
k=\frac{Z}{\nu+n} \Rightarrow E_{n l}=-\frac{1}{2} \frac{Z^{2}}{(n+\nu)^{2}},
$$

where $n=1,2,3, \ldots$ is the iteration number used by AIM, along with the corresponding conditions on the potential parameter $\beta$ reported in Table III

The asymptotic iteration method can also be used to compute approximate eigenvalues of Schrödinger equation (44) for different explicit values of the potential parameters $Z$ and $\beta$. We use $\lambda_{0}$ and $s_{0}$ given by (45), and the numerical results, including the iteration number and suitable $r_{0}$, are reported in Table (IV). It is important to note for the case of $\beta=200$, the eigenvalues for $l=0,1,2,3$ are squeezed together in space less than 0.001 , and this makes it hard for AIM to function correctly. In such cases, it is therefore recommended to use the scaling law as given by (2). For example, we know using the scaling law to show that

$$
E(1,200,2)=\frac{1}{4^{2}} E(4,50,2) .
$$

Thus instead of computing $E(1,200,2)$ we may compute $E(4,50,2)$ then divide the result by 16 . Indeed, for $l=2$ and $l=3$, AIM yields for $E(4,40,2)$, the values -0.0624031366 and -0.0579816331 , respectively. From which we can now compute the eigenvalues $E(1,200,2)$ as reported in Table (IV). 
TABLE III: Polynomial conditions on the potential parameters for the existence of exact solutions of the radial Schrödinger equation (44) for $q=2$.

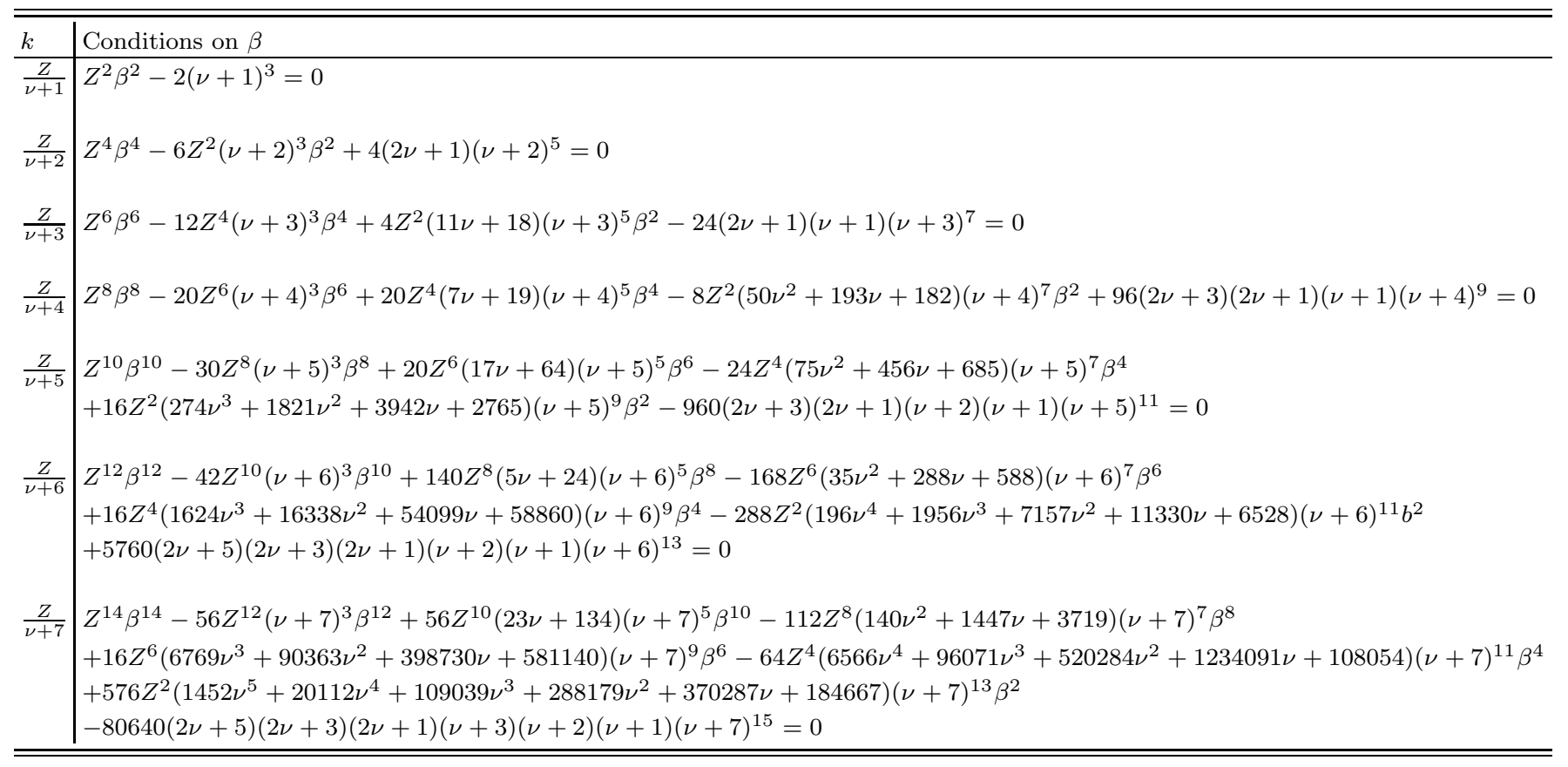

TABLE IV: Approximate solutions of the radial Schrödinger equation (13) for $q=2$. The values marked ${ }^{\dagger}$ are computed using a scaling law, as discussed in the text.

\begin{tabular}{|c|c|c|c|c|}
\hline$\beta$ & $l$ & Energy & $N$ & $r_{0}$ \\
\hline \multirow[t]{4}{*}{200} & 0 & -0.0045028546 & 23 & 3 \\
\hline & 1 & -0.0041930713 & 21 & 3 \\
\hline & 2 & $-0.0039001960^{\dagger}$ & 16 & 2 \\
\hline & 3 & $-0.0036238521^{\dagger}$ & 16 & 2 \\
\hline \multirow[t]{4}{*}{100} & 0 & -0.0086297774 & 21 & 3 \\
\hline & 1 & -0.0078001328 & 18 & 3 \\
\hline & 2 & -0.0070351932 & 21 & 3 \\
\hline & 3 & -0.0063327290 & 19 & 3 \\
\hline \multirow[t]{4}{*}{50} & 0 & -0.0162607212 & 23 & 3 \\
\hline & 1 & -0.0140883751 & 19 & 3 \\
\hline & 2 & -0.0121587115 & 22 & 3 \\
\hline & 3 & -0.0104584212 & 19 & 3 \\
\hline \multirow[t]{4}{*}{35} & 0 & -0.0223342842 & 23 & 3 \\
\hline & 1 & -0.0188109544 & 23 & 3 \\
\hline & 2 & -0.0157614804 & 22 & 3 \\
\hline & 3 & -0.0131524408 & 21 & 3 \\
\hline \multirow[t]{4}{*}{20} & 0 & -0.0361985457 & 25 & 3 \\
\hline & 1 & -0.0288301152 & 24 & 3 \\
\hline & 2 & -0.0227871585 & 23 & 3 \\
\hline & 3 & -0.0179286118 & 24 & 3 \\
\hline \multirow[t]{4}{*}{10} & 0 & -0.0637389182 & 27 & 3 \\
\hline & 1 & -0.0461990390 & 27 & 3 \\
\hline & 2 & -0.0331585888 & 32 & 4 \\
\hline & 3 & -0.0238067362 & 37 & 5 \\
\hline
\end{tabular}




\section{A. Analytic solutions for $V_{2}(r)=-Z / \sqrt{r^{2}+\beta^{2}}$ and the generalized Heun equation}

To analyze the analytic solutions of the differential equation (44), we first let $\chi=\beta z$. We then have

$$
f^{\prime \prime}=\left[2 k \beta+\frac{1}{z}-\frac{(v+1 / 2)}{z-1}-\frac{(v+1 / 2)}{z+1}\right] f^{\prime}+\left[\frac{(\nu+1 / 2) k \beta-Z \beta+k^{2} \beta^{2} / 2}{z-1}+\frac{(\nu+1 / 2) k \beta-Z \beta-k^{2} \beta^{2} / 2}{z+1}-\frac{k \beta}{z}\right] f
$$

or, in the more appropriate form,

$$
f^{\prime \prime}=\left[2 k \beta+\frac{1}{z}-\frac{(v+1 / 2)}{z-1}-\frac{(v+1 / 2)}{z+1}\right] f^{\prime}+\left[\frac{\beta k+\beta^{2} k^{2} z+2 \beta(k \nu-Z) z^{2}}{z(z-1)(z+1)}\right] f .
$$

This equation can be regarded as a special case of the generalized Heun differential equation. According to Schäfke and Schmidt [40], the generalized Heun equation has the form

$$
f^{\prime \prime}+\left[\alpha+\frac{1-\mu_{0}}{z}+\frac{1-\mu_{1}}{z-1}+\frac{1-\mu_{2}}{z-\hat{\mathrm{a}}}\right] f^{\prime}+\left[\frac{\beta_{0}+\beta_{1} z+\beta_{2} z^{2}}{z(z-1)(z-\hat{\mathrm{a}})}\right] f=0
$$

in which $\hat{\mathrm{a}} \in C \backslash\{0,1\}$ and $\mu_{0}, \mu_{1}, \mu_{2}, \alpha, \beta_{0}, \beta_{1}, \beta_{2}$ are arbitrary complex numbers. For $\alpha \neq 0$, the generalized Heun equation (49) has three regular singular points, at 0,1 , â with exponents (i.e. the roots of the indicial equations) $\left\{0, \mu_{0}\right\},\left\{0, \mu_{1}\right\}$ and $\left\{0, \mu_{2}\right\}$, respectively, in addition to one irregular singular point at infinity. These can be shown to be given by:

1. For the singularity $z=0$, we have

$$
\begin{aligned}
\lim _{z \rightarrow 0} z\left(\alpha+\frac{1-\mu_{0}}{z}+\frac{1-\mu_{1}}{z-1}+\frac{1-\mu_{2}}{z-\hat{\mathrm{a}}}\right) & =1-\mu_{0} \\
\lim _{z \rightarrow 0} z^{2}\left(\frac{\beta_{0}+\beta_{1} z+\beta_{2} z^{2}}{z(z-1)(z-\hat{\mathrm{a}})}\right) & =0
\end{aligned}
$$

and the indicial equation then reads

$$
s(s-1)+\left(1-\mu_{0}\right) s=0 \Rightarrow s\left(s-\mu_{0}\right)=0
$$

with simple roots $\left\{0, \mu_{0}\right\}$.

2. For the singularity $z=1$, we have

$$
\begin{aligned}
\lim _{z \rightarrow 1}(z-1)\left(\alpha+\frac{1-\mu_{0}}{z}+\frac{1-\mu_{1}}{z-1}+\frac{1-\mu_{2}}{z-\hat{\mathrm{a}}}\right) & =1-\mu_{1} \\
\lim _{z \rightarrow 1}(z-1)^{2}\left(\frac{\beta_{0}+\beta_{1} z+\beta_{2} z^{2}}{z(z-1)(z-\hat{\mathrm{a}})}\right) & =0
\end{aligned}
$$

and the indicial equation then reads

$$
s(s-1)+\left(1-\mu_{1}\right) s=0 \Rightarrow s\left(s-\mu_{1}\right)=0
$$

with simple roots $\left\{0, \mu_{1}\right\}$.

3. For the singularity $z=\hat{\mathrm{a}}$, we have

$$
\begin{aligned}
\lim _{z \rightarrow \hat{\mathrm{a}}}(z-\hat{\mathrm{a}})\left(\alpha+\frac{1-\mu_{0}}{z}+\frac{1-\mu_{1}}{z-1}+\frac{1-\mu_{2}}{z-\hat{\mathrm{a}}}\right) & =1-\mu_{2} \\
\lim _{z \rightarrow \hat{\mathrm{a}}}(z-\hat{\mathrm{a}})^{2}\left(\frac{\beta_{0}+\beta_{1} z+\beta_{2} z^{2}}{z(z-1)(z-\hat{\mathrm{a}})}\right) & =0
\end{aligned}
$$

and the indicial equation then reads

$$
s(s-1)+\left(1-\mu_{2}\right) s=0 \Rightarrow s\left(s-\mu_{2}\right)=0
$$

with simple roots $\left\{0, \mu_{2}\right\}$. 
To find the exponent of the singular point at $\infty$, we substitute $z=\frac{1}{z_{1}}, z_{1}=\frac{1}{z}, d z_{1}=-\frac{1}{z^{2}} d z$. Further

$$
\frac{d f}{d z}=\frac{d f}{d z_{1}} \frac{d z_{1}}{d z}=-\frac{1}{z^{2}} \frac{d f}{d z_{1}}=-z_{1}^{2} \frac{d f}{d z_{1}}
$$

and

$$
\frac{d^{2} f}{d z^{2}}=\frac{d}{d z} \frac{d f}{d z}=\frac{d}{d z_{1}}\left(-z_{1}^{2} \frac{d f}{d z_{1}}\right) \frac{d z_{1}}{d z}=\left(-2 z_{1} \frac{d f}{d z_{1}}-z_{1}^{2} \frac{d^{2} f}{d z_{1}^{2}}\right)\left(-z_{1}^{2}\right)=2 z_{1}^{3} \frac{d f}{d z_{1}}+z_{1}^{4} \frac{d^{2} f}{d z_{1}^{2}}
$$

Substituting this in (49), we obtain

$$
2 z_{1}^{3} \frac{d f}{d z_{1}}+z_{1}^{4} \frac{d^{2} f}{d z_{1}^{2}}+\left[\alpha+\left(1-\mu_{0}\right) z_{1}+z_{1}\left(\frac{1-\mu_{1}}{1-z_{1}}\right)+z_{1} \frac{1-\mu_{0}}{1-\hat{\mathrm{a}} z_{1}}\right]\left(-z_{1}^{2} \frac{d f}{d z_{1}}\right)+\left(\frac{\beta_{0} z_{1}^{2}+\beta_{1} z_{1}+\beta_{2}}{z_{1}\left(1-z_{1}\right)\left(1-\hat{\mathrm{a}} z_{1}\right)}\right) f=0 .
$$

Thus we have for $\alpha \neq 0$ that $z=\infty$ is an irregular singularity of Poincaré rank 1 , since the term $z_{1}^{k+1}\left(\alpha / z_{1}^{2}\right)=\alpha \neq 0$ for $k=1$. Clearly then for the Heun equation (49) with $\alpha=0$, the singular points 0 , 1 , â and infinity are regular singular points. This is the main difference between the Heun differential equation $(\alpha=0)$ and the generalized Heun equation as given by (49).

In order to find a power series solution of (49) about the singular point $z=0$, i.e. valid for $|z|<1$, we notice first that equation (49) can be written as

$$
\begin{aligned}
\left(z^{3}-(1+b) z^{2}+\hat{a} z\right) f^{\prime \prime} & +\left[\alpha z^{3}+\left(3-\alpha(1+\hat{a})-\mu_{0}-\mu_{1}-\mu_{2}\right) z^{2}+\left(-2+\mu_{0}+\mu_{2}+\left(-2+\mu_{0}+\mu_{1}\right) \hat{a}+\alpha \hat{a}\right) z\right. \\
+ & \left.\left(1-\mu_{0}\right) \hat{a}\right] f^{\prime}+\left[\beta_{0}+\beta_{1} z+\beta_{2} z^{2}\right] f=0 .
\end{aligned}
$$

Using

$$
f(z)=\sum_{k=0}^{\infty} c_{k} z^{k}, \quad \frac{d f}{d z}=\sum_{k=1}^{\infty} k c_{k} z^{k-1}, \quad \frac{d^{2} f}{d z^{2}}=\sum_{k=2}^{\infty} k(k-1) c_{k} z^{k-2}
$$

and substitute in (51), we obtain, after some simplification,

$$
\begin{aligned}
& \sum_{k=3}^{\infty}(k-1)(k-2) c_{k-1} z^{k}-(1+\hat{\mathrm{a}}) \sum_{k=2}^{\infty} k(k-1) c_{k} z^{k}+\hat{\mathrm{a}} \sum_{k=1}^{\infty} k(k+1) c_{k+1} z^{k}+\alpha \sum_{k=3}^{\infty}(k-2) c_{k-2} z^{k} \\
& \quad+\left(3-\alpha(1+\hat{\mathrm{a}})-\mu_{0}-\mu_{1}-\mu_{2}\right) \sum_{k=2}^{\infty}(k-1) c_{k-1} z^{k}+\left(-2+\mu_{0}+\mu_{2}+\left(-2+\mu_{0}+\mu_{1}\right) \hat{\mathrm{a}}+\alpha \hat{\mathrm{a}}\right) \sum_{k=1}^{\infty} k c_{k} z^{k} \\
& \quad+\left(1-\mu_{0}\right) \hat{\mathrm{a}} \sum_{k=0}^{\infty}(k+1) c_{k+1} z^{k}+\beta_{0} \sum_{k=0}^{\infty} c_{k} z^{k}+\beta_{1} \sum_{k=1}^{\infty} c_{k-1} z^{k-1}+B_{2} \sum_{k=2}^{\infty} c_{k-2} z^{k}=0
\end{aligned}
$$

from which we obtain the four-term recurrence relation

$$
\begin{array}{r}
{\left[(k-1)(k-2)+\left(3-\alpha(1+\hat{\mathrm{a}})-\mu_{0}-\mu_{1}-\mu_{2}\right)(k-1)+B_{1}\right] c_{k-1}} \\
+\left[-k(k-1)(1+\hat{\mathrm{a}})+k\left(-2+\mu_{0}+\mu_{2}+\left(-2+\mu_{0}+\mu_{1}\right) \hat{\mathrm{a}}+\alpha \hat{\mathrm{a}}\right)+B_{0}\right] c_{k} \\
+\left[\hat{\mathrm{a}} k(k+1)+\hat{\mathrm{a}}(k+1)\left(1-\mu_{0}\right)\right] c_{k+1}+\left[\alpha(k-2)+B_{2}\right] c_{k-2}=0 .
\end{array}
$$

Replacing $k$ by $k-1$, we obtain

$$
\begin{aligned}
\hat{\mathrm{a} k} k\left(k-\mu_{0}\right) c_{k} & +\left[(k-1)\left(-(k-2)(1+\hat{\mathrm{a}})-2+\mu_{0}+\mu_{2}+\left(-2+\mu_{0}+\mu_{1}\right) \hat{\mathrm{a}}+\alpha \hat{\mathrm{a}}\right)+B_{0}\right] c_{k-1} \\
& +\left[(k-2)\left(k-\alpha(1+\hat{\mathrm{a}})-\mu_{0}-\mu_{1}-\mu_{2}\right)+B_{1}\right] c_{k-2}+\left[\alpha(k-3)+B_{2}\right] c_{k-3}=0
\end{aligned}
$$

with $c_{-1}=c_{-2}=0$. It should be noted that the Floquet solutions of Schäfke and Schmidt [40] to the generalized Heun equation (49) follows by using

$$
c_{k}=\frac{\tau_{k}\left(\mu_{0}, \mu_{1}, \mu_{2}, \alpha, B_{0}, B_{1}, B_{2} ; \hat{a}\right)}{\Gamma\left(k+1-\mu_{0}\right) \Gamma(k+1)}, \quad \tau_{-1}=\tau_{-2}=0
$$


to obtain, using (53), the four-term recurrence relation

$$
\begin{aligned}
\hat{\mathrm{a}} \tau_{k} & =\left[(k-1)\left(k-\mu_{0}-\mu_{2}+\left(k-\mu_{0}-\mu_{1}\right) \hat{\mathrm{a}}-\alpha \hat{\mathrm{a}}\right)-B_{0}\right] \tau_{k-1} \\
& +\left[(k-2)\left(-k+\alpha(\hat{\mathrm{a}}+1)+\mu_{0}+\mu_{1}+\mu_{2}\right)-B_{1}\right](k-1)\left(k-1-\mu_{0}\right) \tau_{k-2} \\
& -\left[\alpha(k-3)+B_{2}\right]\left(k-2-\mu_{0}\right)\left(k-1-\mu_{0}\right)(k-1)(k-2) \tau_{k-3} .
\end{aligned}
$$

That is to say, in the notation of Schäfke and Schmidt,

$$
\tau_{k}=\phi_{1}(k-1) \tau_{k-1}-\phi_{2}(k-2) \tau_{k-2}+\phi_{3}(k-3) \tau_{k-3}
$$

where

$$
\begin{aligned}
& \phi_{1}(\xi)=\xi\left(\xi+1-\mu_{0}-\mu_{1}\right)+\frac{1}{\hat{\mathrm{a}}} \xi\left(\xi+1-\mu_{0}-\mu_{2}\right)-\alpha \xi-\frac{B_{0}}{\hat{\mathrm{a}}} \\
& \phi_{2}(\xi)=(\xi+1)\left(\xi+1-\mu_{0}\right)\left(\frac{1}{\hat{\mathrm{a}}} \xi\left(\xi+2-\mu_{0}-\mu_{1}-\mu_{2}\right)-\left(1+\frac{1}{\hat{\mathrm{a}}}\right) \alpha \xi+\frac{B_{1}}{\hat{\mathrm{a}}}\right) \\
& \phi_{3}(\xi)=-\frac{1}{\hat{\mathrm{a}}}\left(\alpha \xi+B_{2}\right)\left(\xi+1-\mu_{0}\right)\left(\xi+2-\mu_{0}\right)(\xi+1)(\xi+2) .
\end{aligned}
$$

The condition for an $N$-degree polynomial solution is equivalent to the equation $c_{N+3}=0$ in (54), i.e.

$$
\alpha N+B_{2}=0
$$

If this condition is satisfied, the recurrence relation

$$
\begin{aligned}
\hat{\mathrm{a}}(k+1)(k+1 & \left.-\mu_{0}\right) c_{k+1}+\left[k\left(-(k-1)(1+\hat{\mathrm{a}})-2+\mu_{0}+\mu_{2}+\left(-2+\mu_{0}+\mu_{1}\right) \hat{\mathrm{a}}+\alpha \hat{\mathrm{a}}\right)+B_{0}\right] c_{k} \\
& +\left[(k-1)\left(k+1-\alpha(1+\hat{\mathrm{a}})-\mu_{0}-\mu_{1}-\mu_{2}\right)+B_{1}\right] c_{k-1}+\left[\alpha(k-2)+B_{2}\right] c_{k-2}=0
\end{aligned}
$$

for $0 \leq k \leq N$ forms a system of $N+1$ homogeneous linear relation in the $N+1$ coefficients $c_{k}$. This system has a non-trivial solution if and only if its determinant $\Delta_{N+1}$, given explicitly in Table $\mathrm{V}$, is zero; that is to say,

$$
\Delta_{N+1}=0 .
$$

Under the simultaneous fulfillment of the two conditions (58) and (60), the solution of the generalized Heun equation (49) reduces to a polynomial of degree $N$. In what follows, we use these conditions to compute the polynomial solutions of the differential equation (48). These results confirm our earlier findings using AIM and reported in Table (III).

Comparing Eq.(48) and Eq.(49), we have

$$
\begin{aligned}
\mu_{0} & =2, \quad \mu_{1}=\frac{1}{2}-\nu, \quad \mu_{2}=\frac{1}{2}-\nu \\
B_{0} & =-\beta k, \quad B_{1}=-\beta^{2} k^{2}, \quad B_{2}=-2 \beta(k \nu-Z) \\
\alpha & =-2 k \beta, \quad \hat{\mathrm{a}}=-1 .
\end{aligned}
$$

Condition (58), immediately yields

$$
k=\frac{Z}{N+\nu} .
$$

The polynomial solutions can be found by means of the condition (60): for $N=1$, we have

$$
\Delta_{2}=\left|\begin{array}{cc}
-\beta^{2} k^{2} & \beta k \\
-2 \beta(k \nu-Z) & 2 \nu-\beta^{2} k^{2}
\end{array}\right|=\beta^{2} k\left(\beta^{2} k^{3}-2 Z\right)=0 .
$$




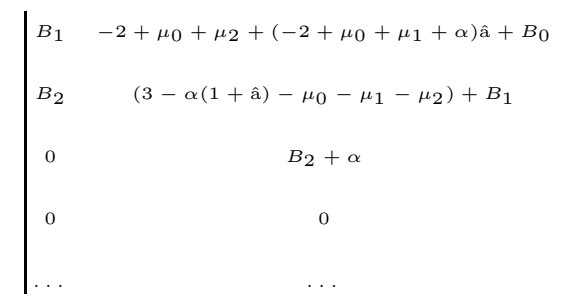

\begin{tabular}{|c|c|c|c|c|}
\hline $2 \hat{a}\left(2-\mu_{0}\right)$ & 0 & 0 & 0 & 0 \\
\hline $2\left(-3+\mu_{0}+\mu_{2}+\left(-3+\mu_{0}+\mu_{1}+\alpha\right) \hat{\mathrm{a}}\right)+B_{0}$ & $3 \hat{a}\left(3-\mu_{0}\right)$ & 0 & 0 & 0 \\
\hline $2\left(4-\alpha(1+\hat{a})-\mu_{0}-\mu_{1}-\mu_{2}\right)+B_{1}$ & $3\left(-4+\mu_{0}+\mu_{2}+\left(-4+\mu_{0}+\mu_{1}+\alpha\right) \hat{a}\right)+B_{0}$ & $4 \hat{a}\left(4-\mu_{0}\right)$ & 0 & 0 \\
\hline$B_{2}+2 \alpha$ & $3\left(5-\alpha(1+\hat{a})-\mu_{0}-\mu_{1}-\mu_{2}\right)+B_{1}$ & $4\left(-5+\mu_{0}+\mu_{2}+\left(-5+\mu_{0}+\mu_{1}+\alpha\right) \hat{a}\right)+B_{0}$ & 0 & 0 \\
\hline
\end{tabular}


From this, for $k=Z /(\nu+1)$, we have

$$
Z^{2} \beta^{2}-2(\nu+1)^{3}=0
$$

with polynomial solution given by

$$
\begin{aligned}
f_{1}(z) & =1+\frac{\beta Z}{\nu+1} z \\
& =1+\frac{Z}{\nu+1} \chi \\
& =1+\frac{Z}{\nu+1} \sqrt{r^{2}+\beta^{2}}
\end{aligned}
$$

For $N=2$, we have

$$
\Delta_{3}=\left|\begin{array}{ccc}
-\beta^{2} k^{2} & \beta k & 0 \\
-2 \beta(k \nu-Z) & 2 \nu-\beta^{2} k^{2} & 3 k \beta \\
0 & -2 \beta(k \nu-Z)-2 \beta k & 2+4 \nu-\beta^{2} k^{2}
\end{array}\right|=0
$$

from which, for $k=Z /(\nu+2)$, we have

$$
Z^{4} \beta^{4}-6 Z^{2}(\nu+2)^{3} \beta^{2}+4(2 \nu+1)(\nu+2)^{5}=0 .
$$

with polynomial solution given by

$$
\begin{aligned}
f_{2}(z) & =1+\frac{Z \beta}{\nu+2} z+\frac{2 \beta^{2} Z^{2}}{\beta^{2} Z^{2}-2(2 \nu+1)(2+\nu)^{2}} z^{2} \\
& =1+\frac{Z}{\nu+2} \chi+\frac{2 Z^{2}}{\beta^{2} Z^{2}-2(2 \nu+1)(2+\nu)^{2}} \chi^{2} \\
& =1+\frac{Z}{\nu+2} \sqrt{r^{2}+\beta^{2}}+\frac{2 Z^{2}}{\beta^{2} Z^{2}-2(2 \nu+1)(2+\nu)^{2}}\left(\sqrt{r^{2}+\beta^{2}}\right)^{2}
\end{aligned}
$$

For $N=3$, we have

$$
\Delta_{4}=\left|\begin{array}{cccc}
-\beta^{2} k^{2} & \beta k & 0 & 0 \\
-2 \beta(k \nu-Z) & 2 \nu-\beta^{2} k^{2} & 3 k \beta & -3 \\
0 & -2 \beta(k \nu-Z)-2 \beta k & 2+4 \nu-\beta^{2} k^{2} & 5 \beta k \\
0 & 0 & -2 \beta(k \nu-Z)-4 \beta k & 6+6 \nu-\beta^{2} k^{2}
\end{array}\right|=0
$$

from which, for $k=Z /(\nu+3)$, we have

$$
Z^{6} \beta^{6}-12 Z^{4}(\nu+3)^{3} \beta^{4}+4 Z^{2}(11 \nu+18)(\nu+3)^{5} \beta^{2}-24(2 \nu+1)(\nu+1)(\nu+3)^{7}=0 .
$$

with polynomial solution given by (note $k=Z /(\nu+3))$

$$
\begin{aligned}
f_{3}(z) & =1+\beta k z-\frac{2 \beta^{2} k\left(\beta^{2} k^{2}-6(\nu+1)\right)(k(\nu+1)-Z)}{\beta^{4} k^{4}+2 k(6 k-5 Z) \beta^{2}+12(\nu+1)(2 \nu+1)} z^{2}+\frac{4 k \beta^{3}(k(1+\nu)-Z)(k(\nu+2)-Z)}{\beta^{4} k^{4}+2 k(6 k-5 Z) \beta^{2}+12(\nu+1)(2 n+1)} z^{3} \\
& =1+k \chi-\frac{2 k\left(\beta^{2} k^{2}-6(\nu+1)\right)(k(\nu+1)-Z)}{\beta^{4} k^{4}+2 k(6 k-5 Z) \beta^{2}+12(\nu+1)(2 \nu+1)} \chi^{2}+\frac{4 k(k(1+\nu)-Z)(k(\nu+2)-Z)}{\beta^{4} k^{4}+2 k(6 k-5 Z) \beta^{2}+12(\nu+1)(2 n+1)} \chi^{3} \\
& =1+k \sqrt{r^{2}+\beta^{2}}-\frac{2 k\left(\beta^{2} k^{2}-6(\nu+1)\right)(k(\nu+1)-Z)}{\beta^{4} k^{4}+2 k(6 k-5 Z) \beta^{2}+12(\nu+1)(2 \nu+1)}\left(\sqrt{r^{2}+\beta^{2}}\right)^{2} \\
& +\frac{4 k(k(1+\nu)-Z)(k(\nu+2)-Z)}{\beta^{4} k^{4}+2 k(6 k-5 Z) \beta^{2}+12(\nu+1)(2 n+1)}\left(\sqrt{r^{2}+\beta^{2}}\right)^{3} .
\end{aligned}
$$

Similarly, higher degree polynomial solutions follow from the determinant $\Delta_{N+1}=0$ given by Table IV. 


\section{CONCLUSION}

The Schrödinger operator $H=-\frac{1}{2} \Delta+V_{q}(r)$ is of considerable interest in atomic physics. In this paper we have shown for the two most important cases $q=1$ and $q=2$, that the bound-state eigenequation $H \psi=E \psi$ can be reduced to the problem of solving the confluent Heun differential equation, or the generalized Heun differential equation. In the present context, the wave function can be written in a form in which analytical solutions with the correct asymptotics involve an unknown factor, which in certain cases can be shown to be a polynomial. A recently established Asymptotic Iteration Method (AIM) has been used to determine this factor, and the corresponding eigenvalue, explicitly in cases where the factor is a polynomial, and to construct accurate approximations in other cases.

\section{Acknowledgments}

Partial financial support of this work under Grant Nos. GP3438 and GP249507 from the Natural Sciences and Engineering Research Council of Canada is gratefully acknowledged by two of us (respectively RLH and NS). KDS acknowledges the Department of Science and Technology, New Delhi for the award of J.C.Bose National Fellowship. KDS and NS are grateful for the hospitality provided by the Department of Mathematics and Statistics of Concordia University, where part of this work was carried out.

\section{Appendix I: Confluent Heun differential equation}

In this appendix we summarize some facts about the confluent Heun differential equation, for more details and further properties, we refer the work of P. P. Fiziev [39] and the references therein. The confluent Heun's differential equation in simplest uniform shape is written as

$$
H^{\prime \prime}+\left(a+\frac{\beta+1}{z}+\frac{\gamma+1}{z-1}\right) H^{\prime}+\left(\frac{\mu}{z}+\frac{\nu}{z-1}\right) H=0 .
$$

The equation has two regular singularities, at $z=0$ and $z=1$, and an irregular singularity at $z=\infty$. The constants $\mu$ and $\nu$ are related to the constants $\alpha, \beta, \gamma, \delta, \eta$ in the accepted notation of the confluent Heun function

$$
H=H e(\alpha, \beta, \gamma, \delta, \eta, z)
$$

as follows

$$
\left\{\begin{array}{l}
\delta=\mu+\nu-\alpha\left(\frac{\beta+\gamma+2}{2}\right), \\
\eta=\frac{\alpha(\beta+1)}{2}-\mu-\left(\frac{\beta+\gamma+\beta \gamma}{2}\right) .
\end{array}\right.
$$

The confluent Heun functions $H e(\alpha, \beta, \gamma, \delta, \eta, z)$ reduces to a polynomial of degree $N \geq 0$ in the variable $z$ if and only if the following two conditions are satisfied:

$$
\left\{\begin{array}{l}
\frac{\delta}{\alpha}+\frac{\beta+\gamma}{2}+N+1=0 \\
\Delta_{N+1}(\mu)=0
\end{array}\right.
$$

where $\Delta_{N+1}(\mu)$ is a three-diagonal determinant given by:

$$
\begin{array}{|ccccccc}
\mu-q_{1} & 1(1+\beta) & 0 & \ldots & 0 & 0 & 0 \\
N \alpha & \mu-q_{2}+\alpha & 2(2+\beta) & \ldots & 0 & 0 & 0 \\
0 & (N-1) \alpha & \mu-q_{3}+2 \alpha & \ldots & 0 & 0 & 0 \\
\vdots & \vdots & \vdots & \ddots & \vdots & \vdots & \vdots \\
0 & 0 & 0 & \ldots & \mu-q_{N-1}+(N-2) \alpha & (N-1)(N-1+\beta) & 0 \\
0 & 0 & 0 & \ldots & 2 \alpha & \mu-q_{N}+(N-1) \alpha & N(N+\beta) \\
0 & 0 & 0 & \ldots & 0 & 1 \alpha & \mu-q_{N+1}+N \alpha
\end{array} \mid
$$


for

$$
q_{n}=(n-1)(n+\beta+\gamma)
$$

Under the simultaneous fulfillment of the two additional conditions (72) the confluent Heun function $\mathrm{He}(\alpha, \beta, \gamma, \delta, \eta, z)$ reduces to a polynomial of degree $N$.

[1] H.A. Bethe and E.E. Salpeter, Quantum Mechanics of One- and Two-Electron Atoms, (Dover Publications, New York 1977).

[2] M. Moshinsky, The Harmonic Oscillator in Modern Physics: From Atoms to Quarks, (Gordon and Breach, London, 1969).

[3] K.D. Sen, V. I. Pupyshev and H.E. Montgomery Jr., Ad. Quantum Chem. 57, 25 (2009).

[4] C.H. Mehta and S.H. Patil , Phys. Rev. A 17, 43 (1978).

[5] S.H. Patil, Phys. Rev. A 24, 2913 (1981).

[6] D. Singh, Y. P. Varshni and R. Dutt, Phys. Rev. A 32, 619 (1985).

[7] H. De Meyer and G. Vanden Berghe, J. Phys. A: Math. Gen. 23, 1323 (1990).

[8] A. Sinha and R. Roychoudhury, J. Phys. A: Math. Gen. 23, 3869 (1990).

[9] F. M. Fernández, J. Phys. A: Math. Gen. 24, 1351 (1991).

[10] R.N. Chaudhuri and M. Mondal, Pramana-J.Phys., 39, 493 (1992).

[11] M. Odeh and O. Mustafa, J. Phys. A: Math. Gen. 33,7013(2000.

[12] O. Mustafa and M. Odeh, J. Phys. B: At. Mol. Opt. Phys. 32,3055 (1999).

[13] C.A.S. Lima and L.C.M. Miranda, Phys. Rev. A 23, 3335 (1981).

[14] J.H. Eberly, Q. Su and J. Javanainen, Phys. Rev. Lett. 62, 881 (1989).

[15] Q. Su and J.H. Eberly, Phys. Rev. A 44, 5997 (1991).

[16] M. Protopapas, C.H. Keitel and P.L. Knight, Rep. Prog. Phys. 60, 389 (1997). References therein.

[17] C.W. Clark, J. Phys. B 30, 2517 (1997).

[18] Y. I. Salamin, S. H. Hu, K. Z. Hatsagortsyan, C. H. Keitel, Phys. Rep. 427, 41 (2006).

[19] R. L. Hall, N. Saad, K. D. Sen, and H. Ciftci, Phys. Rev. A 80, 032507 (2009).

[20] M. Reed and B. Simon, Methods of modern mathematical physics II: Fourier analysis and self-adjointness, (Academic Press, New york, 1975). [The operator inequality is proved on p 169].

[21] S. J. Gustafson and I. M. Sigal, Mathematical concepts of quantum mechanics, (Springer, New York, 2006). [The operator inequality is proved for dimensions $d \geq 3$ on page 32.]

[22] R. L. Hall, Phys. Rev. D 22,2062 (1980).

[23] R. L. Hall, J. Math. Phys. 24, 324 (1983).

[24] R. L. Hall, J. Math. Phys. 25, 2708 (1984).

[25] R. L. Hall, J. Math. Phys. 34, 2779 (1993).

[26] H. Ciftci, R.L. Hall and N. Saad, J. Phys. A: Math. Gen.36 11807-11816 (2003).

[27] B. Champion, R. L. Hall, and N. Saad, Int. J. Mod. Phys. A 23 1405-1415 (2008).

[28] M. F. Fernández, J. Phys. A: Math. Gen. 376173 (2004).

[29] H. Ciftci, R.L. Hall and N. Saad, J. Phys. A: Math. Gen.38 1147-1155 (2005).

[30] H. Ciftci, R.L. Hall and N. Saad, Phys. Lett. A 340 388-396 (2005).

[31] T. Barakat, Phys. Lett. A 344411 (2005).

[32] T. Barakat, K. Abodayeh and A. Mukheimer, J. Phys. A: Math. Gen. 381299 (2005).

[33] T. Barakat, J. Phys. A: Math. Gen. 39 823-831 (2006).

[34] O. Bayrak and I. Boztosun I, J. Phys. A: Math. Gen. 396955 (2006).

[35] N. Saad, R.L. Hall and H. Ciftci, J. Phys. A: Math. Gen. 39 8477-8486 (2006).

[36] N. Saad, R.L. Hall and H. Ciftci, J. Phys. A: Math. Gen. 40 10903-10914 (2007).

[37] P. P. Ray and K. Mahata, J. Phys. A: Math. Gen. 22, 3161 (1989).

[38] F. M. Arscott, Periodic Differential equations, (Oxford: Pergamon Press, 1964). The result is a consequence of Lemma 1, page 21 .

[39] P. P. Fiziev, arXiv:0904.0245 (2009).

[40] R. Schäfke and D. Schmidt, SIAM J. Math. Anal. 11 (1980) 848. 